\title{
More than Ophelia syndrome: Multiple paraneoplastic syndromes in pediatric Hodgkin lymphoma
}

\author{
Uğur Demirsoy¹, Burcu Alparslan², Mehmet Celal Şen³, Yonca Anık², Gür Akansel², \\ Gözde Görür4, Bora Gürel ${ }^{5}$, Görkem Aksu6 ${ }^{6}$, Funda Çorapcıoğlu1 \\ Departments of ${ }^{1}$ Pediatric Oncology, ${ }^{2}$ Radiology, ${ }^{3}$ Pediatric Surgery, ${ }^{4}$ Nuclear Medicine, ${ }^{5}$ Pathology and ${ }^{6}$ Radiation Oncology, \\ Kocaeli University Faculty of Medicine, Kocaeli, Turkey. E-mail: udemirsoy@yahoo.com \\ Received: 2nd January 2018, Accepted: 27th January 2018
}

\begin{abstract}
SUMMARY: Demirsoy U, Alparslan B, Şen MC, Anık Y, Akansel G, Görür G, Gürel B, Aksu G, Çorapçığlu F. More than Ophelia syndrome: Multiple paraneoplastic syndromes in pediatric Hodgkin lymphoma. Turk J Pediatr 2019; 61: 139-141.

Paraneoplastic syndromes in Hodgkin lymphoma (HL) can be seen with different symptoms and organ findings within a significant time before definite diagnosis of the primary disease. Achalasia, Holmes-Adie pupil, and limbic encephalitis are rarely reported paraneoplastic components in pediatric HL. In this report, we present an 11-year-old girl who had all these three paraneoplastic components synchronously before HL was identified.
\end{abstract}

Key words: achalasia, Hodgkin lymphoma, Holmes-Adie pupil, limbic encephalitis, paraneoplastic syndrome.

Hodgkin lymphoma (HL), with prominent advances on the way toward identification of both pathogenesis and biology of the disease, is still not totally understood. Paraneoplastic syndromes (PNS) in HL can show up with various symptoms and different organ findings and some of them may regress after treatment of HL. ${ }^{1}$ Achalasia, Holmes-Adie pupil, and limbic encephalitis are rarely reported as paraneoplastic components of pediatric HL. ${ }^{2,3}$ Hodgkin lymphoma with accompanying limbic encephalitis is named 'Ophelia syndrome'. Herein, we report a child who had achalasia first, developed several neurological symptoms afterwards and finally was diagnosed with HL.

\section{Case Report}

An eleven-year-old girl was referred in our pediatric outpatient clinic with complaints of loss of balance with laughter, dizziness, excessive sleeping, behavioral changes and bilateral low eye lids. She had a history of difficulty in swallowing both fluids and solid food that occurred 12 months before which ended up with diagnosis of achalasia (Fig. 1). Heller myotomy procedure was performed in another hospital 4 months after diagnosis but her symptoms persisted despite surgery. A few weeks after surgery, she started to sleep for more than 12 hours at night, only woke up for meals and slept excessively at school even during school breaks at daytime. She stopped communicating with other children and playing games. She was evaluated by a pediatric psychiatrist but no specific diagnosis was made. She also had ocular accommodative symptoms and blurry vision while looking at close up objects for the last few months and was diagnosed with Holmes-Adie pupil after ophthalmologic examination (bilateral dilated pupils unresponsive to light stimulation and proven increased sensitivity response to diluted pilocarpine). She was prescribed corrective eye-glasses.

On physical examination, she was conscious and cooperative with normal motor functions. Auscultation revealed normal lung and heart sounds and there was no hepatosplenomegaly. All cranial nerves were functional and no pyramidal, cerebellar pathology was recognized. There was bilateral ptosis. She had multiple non-tender, enlarged lymph nodes (maximum diameter: $3 \mathrm{~cm}$ ) on right anterior, infracervical and submandibular region. Magnetic resonance imaging (MRI) of the neck revealed a significant conglomeration of lymphadenopathies on the 


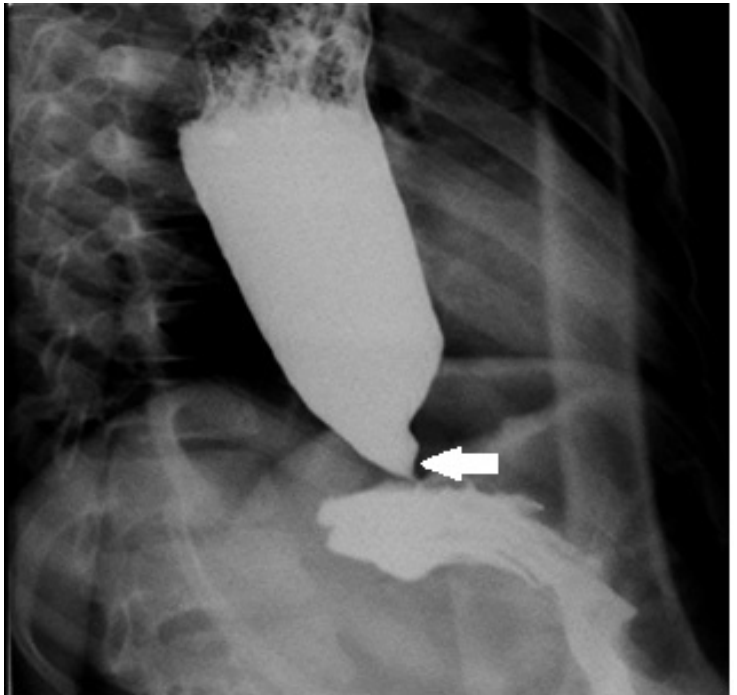

Fig. 1. Barium swallow graphy shows a dilated esophagus with beaking of the lower end (arrow).

right side. Lymph node biopsy of cervical lymph nodes provided a diagnosis of $\mathrm{HL}$, mixed cellular subtype.

Subsequently, the patient underwent a chest computerized tomography (CT) and a fluorodeoxyglucose-positron emission tomography (FDG-PET) scan. Chest CT showed a small number of pulmonary nodules that were less active on the FDG-PET when compared to the lymphadenopathies on the right side of the neck. Also noted on the chest CT was a dilated esophagus, with no FDG uptake. No evidence for external compression was seen to explain esophageal dilation and a tentative diagnosis of achalasia was made. Bone marrow biopsy revealed no pathology. Her disease was classified as stage IV EA considering lung involvement with no accompanying B symptoms.

Since the initial neurological findings persisted, another cranial MRI was performed, which revealed bilaterally increased signal of the hippocampi on FLAIR images, suggesting the diagnosis of limbic encephalitis (Fig. 2). Cerebrospinal fluid (CSF) was clear with no cells and normal protein and glucose levels. Achalasia, limbic encephalitis and Holmes-Adie pupil were considered as components of a PNS of HL. We did not have the opportunity to detect onconeural antibodies in CSF.

Chemotherapy (ABVD: adriamycin $25 \mathrm{mg} / \mathrm{m}^{2}$, bleomycin $15 \mathrm{mg} / \mathrm{m}^{2}$, vinblastin $6 \mathrm{mg} / \mathrm{m}^{2}$,

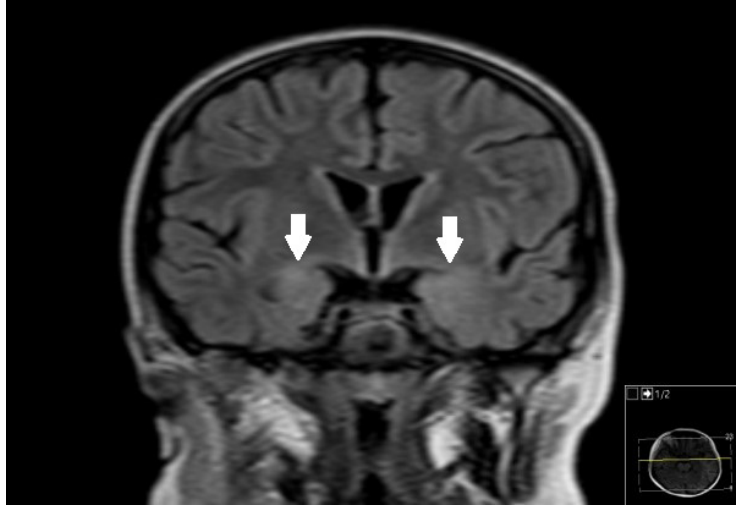

Fig. 2. Coronal FLAIR image shows bilateral symmetric increased signal of the hippocampi (arrows).

dacarbazine $375 \mathrm{mg} / \mathrm{m}^{2}$, on days 0 and 14) was initiated. Interim 18F-FDG PET/CT scan showed complete response after 2 cycles of ABVD. Complaints of dysphagia and bilateral ptosis regressed after four cycles of ABVD. Ocular symptoms and radiological findings of limbic encephalitis (excessive sleeping, loss of balance during laughter) disappeared but esophageal dilation persisted after six cycles of ABVD. She no longer needed her eye-glasses with full visual acuity. Her mother described her feeding as 'slow but comfortable' and defined a significant improvement. Radiotherapy was given to bulky disease. She is still in complete remission for 11 months after the end of treatment.

An informed consent was received from the family before this report was prepared.

\section{Discussion}

Paraneoplastic syndromes can be roughly described as nonmetastatic systemic effects of the primary tumor. These effects have been reported almost in all organ systems, most commonly endocrine, dermatologic, musculoskeletal, and neurological. ${ }^{4}$ Paraneoplastic neurological syndromes rarely associate with Hodgkin lymphoma. It is projected that, after triggering by ectopically expressed antigens by the tumor, misdirected immune responses occur against antigens that are normally present in the nervous system. Paraneoplastic cerebellar degeneration and limbic encephalitis are mostly reported paraneoplastic neurological syndromes in HL patients. In 1982, after a tragic experience with his own daughter (Super Jane) who 
suffered from symptoms of limbic encephalitis without any accurate diagnosis months before identification of HL, Pathologist Dr Carr ${ }^{5}$ proposed this association to be called 'Ophelia Syndrome' regarding the murky character in Shakespeare's play Hamlet. Fifty per cent of HL patients with limbic encephalitis are reported to have seizures, but our case did not so, with a normal electroencephalogram. Super Jane recovered limbic encephalitis after chemotherapy against HL, as also our patient did. Dr Carr mentioned dialysis as an occasionally relieving treatment and related this positive outcome with probable existence of circulating neurotransmitter-like molecule produced by the neoplasm, but they did not practice it for his daughter. Recently, it has been shown that onconeural antibodies (mGluR5, Tr: defined onconeural antibodies in HL) are absent in most PNSs of HL. ${ }^{1}$

Though rare; achalasia is reported in HL. 4,6 A recent case report from India points at a child with Hodgkin lymphoma who presented with achalasia cardia, and Holmes-Adie pupil occurring synchronously with the cancer. ${ }^{3} \mathrm{He}$ had pneumatic dilatation treatment twice, had better esophageal function afterwards but no recovery in bilaterally dilated pupils and his visual acuity remained the same despite chemotherapy. Our patient started to swallow efficiently (without radiological recovery) which he couldn't succeed after surgery and has full acuity after chemotherapy.

Our case is probably the first case in English literature with all these 3 paraneoplastic components in HL. Symptoms of PNS may occur several months before overt findings of $\mathrm{HL}$, and it did happen the same in our case as well (achalasia occured one year before HL). Unsurprisingly, our patient was evaluated by several doctors in different subspecialties (a pediatric surgeon, a pediatric gastroenterologist, an ophthalmologist, a pediatric psychiatrist, and a pediatric neurologist) long before pediatric oncologists. A significant amount of time was spent before diagnosis in most of the reported HL cases. PNS in HL should be kept in mind by all doctors regardless of specialties and patients with vague symptoms should be followed with patience considering PNS.

\section{REFERENCES}

1. Graus F, Ariño H, Dalmau J. Paraneoplastic neurological syndromes in Hodgkin and non-Hodgkin lymphomas. Blood 2014; 123: 3230-3238.

2. Juneja M, Kaur S, Mishra D, Jain S. Ophelia syndrome: Hodgkin lymphoma with limbic encephalitis. Indian Pediatr 2015; 52: 335-336.

3. Srinivasan A, Satish G, Scott JX, Madhavan R, Sathiyasekaran M. Two uncommon paraneoplastic neurological syndromes in a child with Hodgkin lymphoma. J Pediatr Hematol Oncol 2016; 38: 473-475.

4. Petersen CL, Hemker BG, Jacobson RD, Warwick AB, Jaradeh SS, Kelly ME. Wilms tumor presenting with Lambert-Eaton myasthenic syndrome. J Pediatr Hematol Oncol 2013; 35: 267-270.

5. Carr I. The Ophelia syndrome: Memory loss in Hodgkin's disease. Lancet 1982; 1: 844-845.

6. Buyukpamukcu M, Buyukpamukcu N, Cevik N. Achalasia of the oesophagus associated with Hodgkin's disease in children. Clin Oncol 1982; 8: 73-76.

7. Peeples WJ, El-Mahdi AM, Rosato FE. Achalasia of the esophagus associated with Hodgkin disease. J Surg Oncol 1979; 11: 213-216.

8. Katzka DA, Farrugia G, Arora AS. Achalasia secondary to neoplasia: A disease with a changing differential diagnosis. Dis Esophagus 2012; 25: 331-336.

9. Smith MA, Altekruse SF, Adamson PC, Reaman GH, Siebel NL. Declining childhood and adolescent cancer mortality. Cancer 2014; 120: 2497-2506. 